\title{
Programa de Rehabilitación y Desempeño Funcional de pacientes con enfermedad de Parkinson Centro Médico Naval “Cirujano Mayor Santiago Távara"
}

\section{Program of Rehabilitation and Functional Performance of Patients with Parkinson Disease Naval Medical Center "Cirujano Mayor Santiago Távara"}

LesLy Díaz-Meyzan « 1,a , Angello Gambini-Díaz ${ }^{1, a}$, Carolina Pérez-Agüero 1,2,b

\section{Filiación y grado académico \\ Universidad Científica del Sur, Lima, Perú. \\ Centro Médico Naval "Cirujano Mayor Santiago Távara". \\ Médico cirujano. \\ Médico geriatra. \\ 10. ORCID iD de LesLy Díaz-Meyzan \\ http://orcid.org/0000-0001-7038-946X \\ (D.) ORCID iD de Angello Gambini-Díaz https://orcid.org/0000-0001-9725-5090 \\ (10) ORCID iD de Carolina Pérez-Agüero htttp://orcid.org/0000-0001-7218-2876 \\ Contribución de los autores \\ LDM: diseño del estudio, recolección, análisis interpretación de los datos, redacción del ma- nuscrito y aprobación de la versión a publicar. \\ AGD: diseño del estudio, recolección e interpre- tación de los datos, aprobación de la versión a publicar.}

CPA: interpretación de los datos, revisión del manuscrito y aprobación de la versión a publicar.

Fuentes de financiamiento

La investigación fue realizada con recursos propios.

Conflictos de interés

Los autores declaran no tener conflicto de interés.

Recibido: 10-02-2021

Aceptado: 20-04-2021

Citar como

Díaz-Meyzan L, Gambini-Díaz A, Perez-Agüero C Programa de Rehabilitación y Desempeño Funcional de pacientes con enfermedad de Parkinson. Rev Peru Cienc Salud. 2021;3(2): 89-96. doi: https:// doi.org/10.37711/rpcs.2021.3.2.306

Correspondencia Lesly Díaz Meyzan Dirección: Calle Ricardo Palma № 553, Urb. Ingeniería. Lima- Perú. Código Postal: 15102 Teléfono: 51-4810838 Email: diaz.meyzan@gmail.com

\section{RESUMEN}

Objetivo. Determinar la asociación existente entre la aplicación del Programa de Rehabilitación (PR) y el Desempeño Funcional (DF) de pacientes con enfermedad de Parkinson en el Centro Médico Naval “Cirujano Mayor Santiago Távara". Métodos. Estudio analítico, longitudinal, de cohortes retrospectivo, en 18 meses. Mediante una muestra de 46 pacientes, en dos cohortes, se determinó el desempeño funcional por dimensiones de actividades básicas (índices de Barthel), fuerza y resistencia (SPPB) y velocidad de marcha. Se usaron cinco valores o categorías para determinar la dependencia, fragilidad y descoordinación. Se determinó la normalidad mediante la prueba Kolmogorov-Smirnov y para el análisis estadístico se empleó la U de MannWhitney, donde se consideró significativo un valor de $p<0,05$. Resultados. El promedio de edad de pacientes fue $79+/-3$ años (rango: 76-82) de la cohorte expuesta y $72+/-3$ años (69-75) de la cohorte no expuesta al PR, $33(71,7 \%)$ fueron varones del total de la muestra. El 54,3\% (25) tuvieron una o más caídas y en el $45,65 \%$ (21) su cuidador era un familiar. Existe asociación directa entre el PR y el DF $(p=0,009)$ y en sus dimensiones: actividades básicas $(p=0,001)$, fuerza y resistencia $(p=0,001)$ y velocidad de marcha $(p=0,001)$. La cohorte o grupo expuesto al PR mejoró en autonomía, independencia, resistencia y equilibrio en relación al grupo no participante del PR. Conclusiones. Existe asociación directa entre el PR y el DF en todas sus dimensiones: actividades básicas, fuerza, resistencia y velocidad de marcha. A mayor duración del PR se observó mejor DF.

Palabras clave: programa; rehabilitación, rendimiento fisico funcional; enfermedad de Parkinson (Fuente: DeCS-BIREME).

\section{ABSTRACT}

Objective. To determine the existing association between the application of the Rehabilitation Program (RP) and the Functional Performance (DF) of patients with Parkinson's disease at the Naval Medical Center "Cirujano Mayor Santiago Távara". Methods: analytical, longitudinal, retrospective cohort study, in 18 months. With a sample of 46 patients, in two cohorts, Functional Performance was determined by dimensions of basic activities (Barthel indices), strength and endurance (SPPB) and gait speed. Five values or categories were used to determine dependency, frailty, and incoordination. Normality was determined using the Kolmogorov-Smirnov test and the Mann-Whitney $U$ was used for statistical analysis, a significant value of $p$ $<0.05$ was considered. Results: The mean age of patients was $79+/-3$ years (range: 76-82) of the exposed cohort and $72+/-3$ years (69-75) of the cohort not exposed to RP, $33(71,7 \%)$ were men of the total sample. $54.3 \%(25)$ had one or more falls and in $45.6 \%$ (21) their caregiver was a relative. There is a direct association between PR and DF $(p=0.009)$ and in their dimensions: basic activities $(p=0.001)$, strength and endurance $(p=0.001)$ and gait speed $(p=0.001)$. The cohort or group exposed to RP improved in autonomy and independence, resistance and balance in relation to the group that did not participate in RP. Conclusions: there is a direct association between PR and DF in all its dimensions: basic activities, strength and endurance, and gait speed. The longer the PR duration, the better DF is shown.

Keywords: program; rehabilitation; program; physical functional performance; functional performance; Parkinson's disease (Source: MeSH - NLM). 


\section{INTRODUCCIÓN}

La Enfermedad de Parkinson (EP) tiene repercusiones emocionales y motoras, con características conducentes a la disminución de la marcha y de actividades comunes ${ }^{(1-3)}$. La EP se encuentra en el cuarto lugar dentro de las enfermedades neurológicas más frecuentes en el mundo, con una prevalencia entre $1 \%$ y $2 \%$ en mayores de 60 años ${ }^{(4,5)}$ y es la segunda causa de discapacidad neurológica después del accidente cerebrovascular ${ }^{(6)}$. En la provincia de Junín, Perú, la EP reporta una prevalencia cruda de 6,75 por 1000 habitantes $^{(7)}$.

La EP es neurodegenerativa, habiéndose propuesto varias teorías para explicar que la causa, desde factores idiopáticos hasta los genéticos y ambientales, con deterioro progresivo de las neuronas y la reducción dopamina, determina el predominio de neurotransmisores excitatorios ${ }^{(1-3,7,8)}$. Los síntomas y signos principales de la EP son la bradicinesia, acinesia, los temblores, la rigidez muscular, inestabilidad postural, alteraciones del equilibrio, marcha festinante, y facie inexpresiva ${ }^{(1-}$ ${ }^{3,7,8)}$. Esta enfermedad se incrementa con la edad ${ }^{(7)}$ y se reporta un ligero predominio en el varón ${ }^{(8)}$.

Ahora bien, existen varias escalas valorativas del enfermo de Parkinson ${ }^{(8)}$. Un estudio encontró que la depresión es el factor más importante afectando la calidad de vida ${ }^{(9)}$. Sin embargo, las terapias no farmacológicas pueden proporcionar mejoras así, el taichí puede mejorar el equilibrio en la EP ${ }^{(10)}$. Un reporte acerca de que la aplicación de un programa de ejercicio en personas con EP mejora la capacidad física a los 12 meses de seguimiento $^{(11)}$.

Los programas de rehabilitación (PR) son tratamientos muy necesarios en la prevención del deterioro físico precoz; pretendiendo mejorar la sintomatología y, por ende, la calidad de vida de pacientes con EP mediante uso de terapias físicas, para devolver capacidades y oportunidades de autonomía en sus actividades ${ }^{(12,13)}$.

En ese contexto, el tratamiento no farmacológico en la EP es de interés profesional, al ser una medida recomendada por especialistas para prevenir el deterioro de la enfermedad y mejorar equilibrio, la movilidad y disminuir la rigidez ${ }^{(10)}$. Sin embargo, no registra con precisión la relación del PR con la autonomía, resistencia y equilibrio que maneja el DF del parkinsoniano.

El desempeño funcional (DF), para este estudio se dimensionó en actividades básicas, fuerza, resistencia y velocidad de marcha, analizados cada uno mediante cinco niveles. El objetivo del presente estudio fue, por consiguiente, determinar la asociación existente entre la aplicación del PR y el DF, en los pacientes con EP en el Centro Médico Naval (CEMENA) "Cirujano Mayor Santiago Távara", de Callao, Perú.

\section{MÉTODOS}

\section{Tipo de estudio}

La investigación es de tipo observacional, analítica, longitudinal, siendo un estudio de cohortes retrospectivos ${ }^{(12,14)}$.

\section{Población y muestra}

La población estuvo conformada por 57 adultos mayores de más de 60 años, con diagnóstico de $\mathrm{EP}$, que recibían control, tratamiento y terapias de EP en el Servicio de Geriatría CEMENA "Cirujano Mayor Santiago Távara", Callao, Perú, entre enero del 2017 y julio del 2018. Para calcular el tamaño de la muestra se usó Epidat versión 3.1, considerando una potencia de $89 \%$ y nivel de confianza al $95 \%$; el riesgo de expuestos fue del $75 \%$ y el riesgo en no expuestos fue del $30 \%$. La razón de expuestos y no expuestos es 1; el valor calculado para cada cohorte fue de $n=23$, (n1 = GE) (n2= Gne).

Se formó la muestra con los siguientes criterios de inclusión: llegar con o sin acompañamiento de un familiar o cuidador, con capacidad de moverse independientemente y no tener otras enfermedades que interfieran con la rehabilitación; se obtuvo una muestra no probabilística intencionada de 46 participantes. Los criterios de exclusión, por su parte, fueron: pacientes retirados por fallecimiento o causas personales, pacientes parkinsonianos severamente afectados en estadios 4 y 5 de la escala de Hoehn y Yahr ${ }^{(15)}$; poseer trastorno cognitivo o psicológico; tener historia clínica incompleta; haber presentado infarto en el mes previo a la evaluación, o tener diagnóstico de fractura o cirugía reciente. Calculó la potencia estadística descrita en procedimientos.

\section{Instrumentos de recolección de datos}

Programa de rehabilitación (PR): un conjunto de actividades terapéuticas desarrolladas para pacientes con Parkinson, a fin que recuperen funciones en sus dimensiones autonomía, fortaleza y agilidad, disminuidas por la enfermedad ${ }^{(16)}$.

El programa de rehabilitación (PR) diseñado en base a principios del aprendizaje psicomotor, orienta los déficits y mejoras motoras, fue desarrollado en 18 meses mediante 150 sesiones de 2 horas cada una, dos veces por semana 
(martes y viernes), usando modalidad grupal. Se dosificó el tiempo del siguiente modo: fase inicial con calentamiento de 10 minutos. Fase fortalecimiento, consistía en 5 minutos de actividades cardiovasculares, 15 minutos de ejercicios de estiramientos, 15 minutos de ejercicios de fuerza, 15 minutos de un entrenamiento funcional, 15 minutos de entrenamiento de marcha bajo señales auditivas y 15 minutos de equilibrio. Fase relajación de 30 minutos, baile de tango y juegos, y fase despedida, consistente en estímulo o premiación de aplausos.

Desempeño funcional (DF): acciones naturales que desarrollan las personas en sus funciones motoras; específicamente en dimensiones de actividades básicas, fuerza, resistencia, y velocidad de marcha; las que alteran la calidad de vida por efectos de la enfermedad de Parkinson ${ }^{(16)}$. Las variables se operacionalizaron para medir el nivel de dependencia que presentan los pacientes.

Índices de Barthel para las actividades básicas diarias ${ }^{(17-19)}$ (se baña solo, se alimenta solo, usa servicios higiénicos, sube y baja escaleras, se viste solo, controla sus deposiciones, controla sus micciones, se traslada y moviliza solo). El Baremo para procesar los datos considera los siguientes rangos de resultados: 0-20 dependencia total; 21-40 dependencia severa; 41-60 dependencia moderada; 61-90 dependencia leve; >90 autonomía e independencia.

Índices de SPPB o Short Physical Performance Battery, para medir fuerza y resistencia (17-19) (camina con seguridad, se levanta y se sienta, mantiene los pies juntos, mantiene su equilibrio, posición semi tándem y tándem); Se considera en el baremo, los siguientes rangos de resultados: 0-3 fragilidad severa; 4-6 fragilidad moderada; 7-9: fragilidad leve; 10-11 fragilidad mínima; $=12$ resistencia.

Índices de velocidad de marcha ${ }^{(17-19)}$ prueba de ejecución, observa distancia de prueba de 4 metros, ejecución por dos veces, se procesaron datos con el siguiente baremo: incapaz de caminar: descoordinación total; < 8,70 m/s: descoordinación severa; 6,21 a 8,70 m/s: alterado, descoordinación moderada; 4,82 a 6,20 $\mathrm{m} / \mathrm{s}$ : descoordinación leve; < 4,82 m/s: equilibrio y coordinación normal ${ }^{(20)}$.

Se consideraron otras variables que conforman las características demográficas de los pacientes de estudio: edad, sexo, peso, número de caídas y si tiene cuidador. El instrumento de recolección de datos utilizado fue la ficha de observación, cuya validez de contenido fue sometida a evaluación de expertos (4 jueces), el puntaje fue de 0,93; mientras que la confiabilidad fue determinada según el coeficiente de Alfa de Cronbach, cuyo valor fue de 0,805.

\section{Procedimientos de la recolección de datos.}

Previa aprobación del Comité de Ética y División de Investigación del CEMENA se recolectaron datos de las historias clínicas en una ficha de registro de datos y la información obtenida fue procesada en forma anónima (21). No hubo consentimiento informado por tratarse de un estudio de cohortes retrospectivos.

\section{Análisis de datos}

El análisis estadístico se realizó con el programa estadístico SPSS versión 24 . Se resume la información de las variables cualitativas según frecuencias absolutas y relativas, y las variables cuantitativas según medias, mediana y desviación estándar. La distribución de los datos numéricos fue anormal según la prueba de Kolmogorov-Smirnov. Para controlar a las variables intervinientes se emparejó a los grupos de estudio según sexo y edad. Para el análisis inferencial se empleó la prueba $U$ de Mann Whitney para determinar la diferencia entre las medias a efectos de contrastar las hipótesis, considerándose significativo $p<0,05$.

\section{Aspectos éticos}

El estudio fue aprobado por el Comité Institucional de Ética en Investigación de la Universidad Científica del Sur; asimismo, se tuvo en cuenta la Declaración de Helsinki ${ }^{(21)}$, principios 9 y 10, tomando precauciones para resguardar la intimidad de personas e historias necesarias en la investigación.

\section{RESULTADOS}

Se incluyeron 46 (100\%) datos de pacientes, el promedio de edad fue $79+/-3$ años (rango: 76-82) de la cohorte expuesta y $72+/-3$ años (69-75) de la cohorte no expuesta al PR. En tabla 1 se muestra mayor proporción de varones $33(71,7 \%)$ y $13(28,3 \%)$ de mujeres. Se observa que los pacientes parkinsonianos adheridos al PR tienen mayor peso en comparación a la cohorte no expuesta al PR. En 23 (50,0 \%) pacientes, el cuidado estaba a cargo de un familiar, 19 (41,3\%) asisten solos y 4 (8,7\%) pacientes están cuidados por técnicos en enfermería. El 34,8 \% (16) no tuvieron caídas, mientras que el 65,2% (30) presentó un episodio o más de caídas; aunque las caídas fueron más frecuentes en el grupo no expuesto al PR 36,9\% (17) en comparación a los expuestos al PR 28,3 \% (13). 
Tabla 1. Características demográficas clínicas de pacientes geriátricos con enfermedad de Parkinson

\begin{tabular}{|c|c|c|c|c|}
\hline \multirow[b]{2}{*}{ Variable } & \multicolumn{2}{|c|}{ Grupo expuesto al Programa de Rehabilitación } & \multicolumn{2}{|c|}{ Grupo no expuesto al Programa de Rehabilitación } \\
\hline & $\begin{array}{l}\text { Basal } \\
\text { n (\%) }\end{array}$ & $\begin{array}{l}\text { Final } \\
\text { n (\%) }\end{array}$ & $\begin{array}{l}\text { Basal } \\
\mathbf{n}(\%)\end{array}$ & $\begin{array}{l}\text { Final } \\
\text { n (\%) }\end{array}$ \\
\hline \multicolumn{5}{|l|}{ Edad (años) } \\
\hline $60-68$ & $6(26,1)$ & $6(26,1)$ & $3(13,0)$ & $4(17,4)$ \\
\hline $69-75$ & $6(26,1)$ & $6(26,1)$ & $12(52,9)$ & $11(47,8)$ \\
\hline $76-82$ & $8(34,8)$ & $7(30,4)$ & $5(21,7)$ & $5(21,7)$ \\
\hline Más de 82 & $3(13,0)$ & $4(17,4)$ & $3(13,1)$ & $3(13,0)$ \\
\hline \multicolumn{5}{|l|}{ Sexo } \\
\hline Masculino & $16(69,6)$ & $16(69,6)$ & $17(73,9)$ & $17(73,9)$ \\
\hline Femenino & $7(30,4)$ & $7(30,4)$ & $6(26,1)$ & $6(26,1)$ \\
\hline \multicolumn{5}{|l|}{ Peso (kg) } \\
\hline 40 a 50 & $1(4,4)$ & $1(4,4)$ & $2(8,7)$ & $3(13,0)$ \\
\hline 51 a 60 & $7(30,4)$ & $6(26,1)$ & $6(26,1)$ & $7(30,4)$ \\
\hline 61 a 70 & $5(21,7)$ & $6(26,1)$ & $7(30,4)$ & $7(30,4)$ \\
\hline Más de 71 & $10(43,5)$ & $10(43,5)$ & $8(34,8)$ & $6(26,1)$ \\
\hline \multicolumn{5}{|l|}{ Cuidador del paciente } \\
\hline No tiene & $9(39,1)$ & $9(39,1)$ & $11(47,8)$ & $10(43,5)$ \\
\hline FamiSliar & $10(43,5)$ & $11(47,8)$ & $12(52,2)$ & $12(52,2)$ \\
\hline Técnico en enfermería & $4(17,4)$ & $3(13,0)$ & $0(0,0)$ & $1(4,4)$ \\
\hline \multicolumn{5}{|l|}{ Número de caídas } \\
\hline 0 & $11(47,8)$ & $10(43,5)$ & $12(52,2)$ & $6(26,1)$ \\
\hline 1 a 3 & $9(39,1)$ & $10(43,5)$ & $9(39,1)$ & $10(43,5)$ \\
\hline 4 a 6 & $3(13,0)$ & $3(13,0)$ & $1(4,4)$ & $3(13,0)$ \\
\hline 7 a más & $0(0,0)$ & $0(0,0)$ & $1(4,4)$ & $4(17,4)$ \\
\hline
\end{tabular}

En la tabla 2 se evidencian las diferencias significativas entre los dos grupos de estudio. Los pacientes expuestos al PR mejoraron su DF, el 8,7 \% (2) ingresaron con deficiencia severa, reduciéndose a 4,4\% (1) al finalizar el PR. Al inicio del PR, el 30,4\% (7) presentaban un nivel basal de autonomía y normalidad; 18 meses después, al finalizar el PR, el 39,1 \% (9) presentaban autonomía y normalidad. Comparativamente, entre quienes no participaron del PR, el 56,5\% (13) iniciaron con autonomía y normalidad, mientras que 18 meses después hubo un descenso y sólo se alcanzó el $21,7 \%$ (5), en este mismo grupo de estudio, la dependencia moderada aumentó en comparación al basal (13,0 \% vs. 43,5 \%). Confirmándose por consiguiente la hipótesis de que la

Tabla 2. Comparativa inicio y final en el desempeño funcional de pacientes geriátricos con enfermedad de Parkinson

\begin{tabular}{|c|c|c|c|c|}
\hline \multirow{2}{*}{ Desempeño funcional } & \multicolumn{2}{|c|}{$\begin{array}{c}\text { Grupo expuesto al Programa de } \\
\text { Rehabilitación }\end{array}$} & \multicolumn{2}{|c|}{$\begin{array}{c}\text { Grupo no expuesto al Programa de } \\
\text { Rehabilitación }\end{array}$} \\
\hline & $\begin{array}{c}\text { BASAL } \\
\text { n (\%) }\end{array}$ & $\begin{array}{l}\text { FINAL } \\
\text { n (\%) }\end{array}$ & $\begin{array}{c}\text { BASAL } \\
\text { n (\%) }\end{array}$ & $\begin{array}{l}\text { FINAL } \\
\text { n (\%) }\end{array}$ \\
\hline Deficiencia total & $0(0,0)$ & $0(0,0)$ & $0(0,0)$ & $1(4,4)$ \\
\hline Deficiencia severa & $2(8,7)$ & $1(4,4)$ & $1(4,4)$ & $4(17,4)$ \\
\hline Deficiencia moderada & $6(26,1)$ & $4(17,4)$ & $3(13,0)$ & $10(43,5)$ \\
\hline Deficiencia leve & $8(34,8)$ & $9(39,1)$ & $6(26,1)$ & $3(13,0)$ \\
\hline Autónomo. Normal & $7(30,4)$ & $9(39,1)$ & $13(56,5)$ & $5(21,7)$ \\
\hline
\end{tabular}

Nota. Estadístico de prueba: $U$ de Mann-Whitney de 146,$000 ; Z=--2,603 ; p=0,009$ 
Tabla 3. Desempeño funcional referente a la dimensión actividades básicas, de pacientes geriátricos con enfermedad de Parkinson

\begin{tabular}{|c|c|c|c|c|c|c|c|c|}
\hline \multirow{3}{*}{$\begin{array}{l}\text { Desempeño funcional } \\
\text { según actividades básicas }\end{array}$} & \multicolumn{8}{|c|}{ Evaluación del programa de rehabilitación } \\
\hline & \multicolumn{2}{|c|}{ Basal } & \multicolumn{2}{|c|}{6 meses } & \multicolumn{2}{|c|}{12 meses } & \multicolumn{2}{|c|}{18 meses } \\
\hline & $\begin{array}{c}\text { Grupo } \\
\text { expuesto } \\
\mathrm{n}(\%)\end{array}$ & $\begin{array}{c}\text { Grupo no } \\
\text { expuesto } \\
n(\%)\end{array}$ & $\begin{array}{c}\text { Grupo } \\
\text { expuesto } \\
\mathrm{n}(\%)\end{array}$ & $\begin{array}{c}\text { Grupo no } \\
\text { expuesto } \\
n(\%)\end{array}$ & $\begin{array}{c}\text { Grupo } \\
\text { expuesto } \\
\mathrm{n}(\%)\end{array}$ & $\begin{array}{c}\text { Grupo no } \\
\text { expuesto } \\
\mathrm{n}(\%)\end{array}$ & $\begin{array}{l}\text { Grupo } \\
\text { expuesto } \\
\text { n (\%) }\end{array}$ & $\begin{array}{c}\text { Grupo no } \\
\text { expuesto } \\
\mathrm{n}(\%)\end{array}$ \\
\hline Dependencia total & $0(0,0)$ & $0(0,0)$ & $0(0,0)$ & $1(4,4)$ & $1(4,4)$ & $1(4,4)$ & $0(0,0)$ & $1(4,4)$ \\
\hline Dependencia severa & $0(0,0)$ & $0(0,0)$ & $0(0,0)$ & $0(0,0)$ & $0(0,0)$ & $0(0,0)$ & $0(0,0)$ & $0(0,0)$ \\
\hline Dependencia moderada & $2(8,7)$ & $3(13,0)$ & $2(8,7)$ & $5(21,7)$ & $0(0,0)$ & $7(30,4)$ & $0(0,0)$ & $8(34,8)$ \\
\hline Dependencia leve & $17(73,9)$ & $13(56,5)$ & $10(43,5)$ & $12(52,2)$ & $12(52,2)$ & $14(60,8)$ & $7(30,4)$ & $11(47,8)$ \\
\hline Independencia & $4(17,4)$ & $7(30,4)$ & $11(47,8)$ & $5(21,7)$ & $10(43,5)$ & $1(4,4)$ & $16(69,6)$ & $3(13,0)$ \\
\hline
\end{tabular}

Nota. Estadístico de prueba U de Mann-Whitney de 64,500; $Z=-4,410 ; p=0,001$

aplicación del PR tiene asociación directa con el DF en pacientes con enfermedad de Parkinson. La prueba $U$ de Mann-Whitney fue de $146,00(Z=-2,603 ; p=0,009)$.

En la tabla 3 se observa que el $73,9 \%$ de los pacientes con enfermedad de Parkinson expuestos al PR inicia con dependencia leve en sus actividades básicas y después de participar durante 18 meses presenta una mejoría, disminuyendo el grupo de dependencia al 30,4\%. Por su parte, el grupo de pacientes no expuestos al PR se encontraba con una dependencia moderada en el $13,0 \%$, después de 18 meses que se encontró un incremento a $34,8 \%$. El grupo expuesto al PR $(17,4 \%)$ consiguió mejoría para desarrollar actividad básica de forma independiente $(69,6 \%)$. Todo lo contrario ocurrió en el grupo no expuesto al PR, evidenciándose empeoramiento en su independencia del $30,4 \%$ a $13,0 \%$. Se concluye que hay razones suficientes para inferir que ambas muestras presentaban diferencias significativas, afirmando por lo tanto que la aplicación del PR tiene resultados favorables en la dimensión actividades básicas. Para analizar los resultados de la aplicación del PR en la dimensión actividades básicas se utilizó la prueba de $U$ de Mann-Whitney que fue de 64,500 ( $Z=-4,410$ у $p=0,001)$.

Parkinson inicialmente expuestos al PR fue del 13,0\%, mientras que al finalizar el PR, el porcentaje de pacientes que mejoraron su fuerza resistencia se incrementó, reportándose en el 30,5\%. Mientras que en el grupo que no asistió al PR los hallazgos fueron diferentes, ya que este grupo había iniciado con cifras similares $(13,0 \%)$ al grupo expuesto al PR, sin embargo al finalizar el estudio, solo el 4,3\% reportaba una resistencia normal. El grupo $\sin$ PR con dependencia severa y moderada empeoró de $4,4 \%$ a $34,8 \%$ y de $43,5 \%$ a $52,2 \%$, respectivamente. Para analizar los resultados de la aplicación del PR en la dimensión fuerza y resistencia, se empleó la prueba de $U$ de Mann-Whitney que fue de 99,500 ( $Z=-3,660$ у $p=0,001$ ).

En la tabla 5 se muestra que el PR está asociado con un mejor desempeño funcional de la velocidad de la marcha a través del tiempo. El grupo de pacientes expuestos

Tabla 4. Desempeño funcional, referente a la dimensión fuerza y resistencia, de pacientes geriátricos con enfermedad de Parkinson 2018

\begin{tabular}{|c|c|c|c|c|c|c|c|c|}
\hline \multirow{3}{*}{$\begin{array}{l}\text { Desempeño funcional } \\
\text { según fuerza y } \\
\text { resistencia }\end{array}$} & \multicolumn{8}{|c|}{ Evaluación del programa de rehabilitación } \\
\hline & \multicolumn{2}{|c|}{ Basal } & \multicolumn{2}{|c|}{6 meses } & \multicolumn{2}{|c|}{12 meses } & \multicolumn{2}{|c|}{18 meses } \\
\hline & $\begin{array}{c}\text { Grupo } \\
\text { expuesto } \\
n(\%)\end{array}$ & $\begin{array}{c}\text { Grupo no } \\
\text { expuesto } \\
\text { n (\%) }\end{array}$ & $\begin{array}{l}\text { Grupo } \\
\text { expuesto } \\
\text { n (\%) }\end{array}$ & $\begin{array}{c}\text { Grupo no } \\
\text { expuesto } \\
\text { n (\%) }\end{array}$ & $\begin{array}{c}\text { Grupo } \\
\text { expuesto } \\
\mathbf{n}(\%)\end{array}$ & $\begin{array}{c}\text { Grupo no } \\
\text { expuesto } \\
\text { n (\%) }\end{array}$ & $\begin{array}{c}\text { Grupo } \\
\text { expuesto } \\
\text { n (\%) }\end{array}$ & $\begin{array}{c}\text { Grupo no } \\
\text { expuesto } \\
n(\%)\end{array}$ \\
\hline Fragilidad severa & $0(0,0)$ & $0(0,0)$ & $0(0,0)$ & $1(4,4)$ & $1(4,4)$ & $1(4,4)$ & $0(0,0)$ & $1(4,4)$ \\
\hline Fragilidad moderada & $6(26,1)$ & $1(4,4)$ & $3(13,0)$ & $3(13,0)$ & $1(4,4)$ & $3(13,0)$ & $1(4,4)$ & $8(34,8)$ \\
\hline Fragilidad leve & $11(47,8)$ & $10(43,5)$ & $11(47,8)$ & $13(56,5)$ & $10(43,5)$ & $17(73,9)$ & $9(39,1)$ & $12(52,2)$ \\
\hline Fragilidad mínima & $3(13,0)$ & $9(39,1)$ & $5(21,7)$ & $5(21,7)$ & $6(26,1)$ & $1(4,4)$ & $6(26,1)$ & $1(4,4)$ \\
\hline Resistencia normal & $3(13,0)$ & $3(13,0)$ & $4(17,4)$ & $1(4,4)$ & $5(21,7)$ & $1(4,4)$ & $7(30,4)$ & $1(4,4)$ \\
\hline
\end{tabular}

Nota. Estadístico de prueba U de Mann-Whitney de 99,500 ( $Z=-3,660$ y $p=0,001)$. 
Tabla 5. Desempeño funcional, referente a la dimensión velocidad de marcha, de pacientes geriátricos con enfermedad de Parkinson

\begin{tabular}{|c|c|c|c|c|c|c|c|c|}
\hline \multirow{3}{*}{$\begin{array}{l}\text { Desempeño funcional según } \\
\text { velocidad de marcha }\end{array}$} & \multicolumn{8}{|c|}{ Evaluación del programa de rehabilitación } \\
\hline & \multicolumn{2}{|c|}{ Basal } & \multicolumn{2}{|c|}{6 meses } & \multicolumn{2}{|c|}{12 meses } & \multicolumn{2}{|c|}{18 meses } \\
\hline & $\begin{array}{c}\text { Grupo } \\
\text { expuesto } \\
\text { n (\%) }\end{array}$ & $\begin{array}{c}\text { Grupo no } \\
\text { expuesto } \\
n(\%)\end{array}$ & $\begin{array}{c}\text { Grupo } \\
\text { expuesto } \\
\text { n (\%) }\end{array}$ & $\begin{array}{c}\text { Grupo no } \\
\text { expuesto } \\
\text { n (\%) }\end{array}$ & $\begin{array}{c}\text { Grupo } \\
\text { expuesto } \\
\text { n (\%) }\end{array}$ & $\begin{array}{c}\text { Grupo no } \\
\text { expuesto n } \\
(\%)\end{array}$ & $\begin{array}{c}\text { Grupo } \\
\text { expuesto } \\
\text { n (\%) }\end{array}$ & $\begin{array}{c}\text { Grupo no } \\
\text { expuesto } \\
n(\%)\end{array}$ \\
\hline Descoordinación total & $0(0,0)$ & $0(0,0)$ & $0(0,0)$ & $1(4,4)$ & $1(4,4)$ & $1(4,4)$ & $0(0,0)$ & $1(4,4)$ \\
\hline Descoordinación severa & $6(26,1)$ & $1(4,4)$ & $3(13,8)$ & $3(13,8)$ & $1(4,4)$ & $3(13,0)$ & $1(4,4)$ & $8(34,8)$ \\
\hline Descoordinación moderada & $11(43,8)$ & $10(43,5)$ & $11(43,8)$ & $13(56,5)$ & $10(43,5)$ & $17(73,9)$ & $9(39,1)$ & $12(52,2)$ \\
\hline Descoordinación leve & $3(13,0)$ & $9(39,1)$ & $5(21,8)$ & $5(21,8)$ & $6(26,1)$ & $1(4,4)$ & $6(26,1)$ & $1(4,4)$ \\
\hline Equilibrio, coordinación normal & $3(13,0)$ & $3(13,0)$ & $4(17,4)$ & $1(4,4)$ & $5(21,7)$ & $1(4,4)$ & $7(30,4)$ & $1(4,4)$ \\
\hline
\end{tabular}

Nota. Estadístico de prueba: $U$ de Mann-Whitney de 22,000 ( $Z=-5,330$ y $p=0,001)$.

al PR, inicialmente presentaron una descoordinación severa en el $26,1 \%$ y moderada en $43,5 \%$, mientras que después de 18 semanas de aplicación del PR, el $4,4 \%$ y el 39,1\% presentaban descoordinación severa y moderada, observándose una mejoría notoria. Por otro lado, en el grupo no expuesto al PR, la descoordinación basal severa fue de 4,4 \% y la moderada fue del $43,83 \%$, reportando la evaluación final en el 34,8 \% severa y en el 52,2 \% moderada. En este grupo de pacientes, el deterioro funcional era mayor. Asimismo, solo el 13,0\% de pacientes con enfermedad de Parkinson inicialmente presentaron equilibrio y coordinación normal en el grupo con intervención del PR y al finalizar la intervención hubo más pacientes que incrementaron el nivel de equilibrio y coordinación normal (30,4\%). Para evaluar los resultados de la aplicación del PR en la dimensión velocidad de marcha se empleó la prueba $U$ de Mann-Whitney cuyo valor fue de 22,000 ( $Z=-5,330$ y $p=0,001)$.

\section{DISCUSIÓN}

La EP es la segunda causa más común de enfermedad neurodegenerativa después de la enfermedad de Alzheimer ${ }^{(22,23)}$; de progreso lento e invalidante tanto física, cognitiva y socialmente, afecta aproximadamente al 0,3\% de la población, incrementándose hasta el 3\% en mayores de 65 años ${ }^{(22,23)}$. La progresión y severidad de la EP es variable entre los pacientes(24). Según JuradoCoronel et al. ${ }^{(25)}$, la EP es más frecuente en hombres que en mujeres en relación aproximada de 2 a 1 . En nuestra serie, la relación fue de 2,5 a 1. Las personas que padecen de EP presentan el doble de posibilidades de experimentar caídas, comparados con personas mayores sanas; esto conlleva a efectos negativos en confianza, niveles de actividad y calidad de vida ${ }^{(26,27)}$. En ese sentido, se encontró que cada 3 pacientes con
EP, 2 sufrieron entre 1 a más caídas. Teniendo mayor número quienes no participaron del PR. Las terapias de apoyo son implementadas por profesionales de la salud, no médicos, tales como los fisioterapeutas, enfermeros, etc. En este estudio se encontró cuidadores técnicos en muy bajo porcentaje. Según Cersosimo et al. ${ }^{(27)}$, la pérdida de peso ocurre en la mitad de los pacientes con EP y, con gran consecuencia, en la progresión de la propia enfermedad con movimientos involuntarios y la disminución de ingesta de alimentos. En este estudio se encontró que pacientes no expuestos al PR tuvieron menos peso.

En pacientes con EP, los programas de rehabilitación han sido propuestos como un tratamiento complementario y efectivo para mejorar las disfunciones posturales y la marcha ${ }^{(28)}$. Hashimoto et al. ${ }^{(29)}$, en su estudio concluye que la danza es útil para mejorar la función motora, afectiva y los síntomas mentales en los pacientes con EP.

La importancia clínica de los programas de rehabilitación en los pacientes con EP está relacionada con diversos criterios, como la efectividad a largo plazo, dados por la frecuencia, seguimiento y duración del tratamiento, o cambios en la calidad de vida ${ }^{(13)}$. Los programas de rehabilitación basados en actividades físicas aún parecen ser una forma más común de rehabilitación, desde caminar hasta programas de ejercicios personalizados ${ }^{(30,31)}$, y es más efectiva cuando se realiza durante varios años ${ }^{(31)}$.

Franciotta et al. ${ }^{(32)}$ reportaron que los pacientes con EP que se sometieron a un PR donde se incluía terapia de manos, mejoraron en la destrezas con los dedos y en la funcionabilidad de las manos. Los PR multidisciplinarios: cognitivo, motor, aeróbico, intensivo y basado en objetivos, podrían mejorar la calidad de vida de los pacientes 
parkinsonianos ${ }^{(16,33)}$. En nuestro estudio, el DF de los pacientes con EP mejoró a través del tiempo, por lo que el PR a que son expuestos brinda beneficios motores.

Seco-Calvo et al. ${ }^{(34)}$ reportan que cuando se administra a los pacientes con EP un programa de fisioterapia grupal, conjuntamente con estímulos sensoriales auditivos para la reeducación de la marcha, se produce una mejoría significativa sobre los trastornos de equilibrio dinámico y la movilidad funcional. Otro estudio demostró la eficiencia de la fisioterapia a largo plazo con mejoría de la calidad de vida en los pacientes con EP ${ }^{(29)}$. Tickle-Degnen et al. ${ }^{(35)}$, con su programa de rehabilitación, reportan beneficios mayores en los dominios específicos de movilidad y comunicación que persistieron a los 2 y 6 meses de seguimiento. Otro estudio reporta que si se incrementa el número de sesiones del programa de rehabilitación, los resultados en el examen motor son más altos, lo que indica que la rigidez, postura, equilibrio y marchamejoran y que esta mejoría es más prolongada en el tiempo ${ }^{(13)}$. En nuestra serie, la marcha mejoró en los pacientes que recibían el PR.

Por último, existen reportes sobre los beneficios de los ejercicios aeróbicos y el entrenamiento de la fuerza en pacientes con EP mejora los síntomas motores y no motores, particularmente entre el estado de ánimo (depresión), sueño y cognición ${ }^{(36)}$. Según Gobbi et al. ${ }^{(37)}$, los programas de ejercicio intensivo y adaptativo medidos a través de la prueba de timed up and go demostraron mejora en el equilibrio y la movilidad en los pacientes con EP, por lo que sería útil incorporarla a futuros PR en el CEMENA.

Las limitaciones del estudio comprenden la escasa muestra, la posibilidad de que exista variabilidad intraobservacional, de la valoración del estado funcional de los pacientes en los registros anotados. Los sesgos de selección disminuyeron porque ambas cohortes extraídas de las historias clínicas permitieron su comparabilidad. Se concluye que el Programa de Rehabilitación tiene resultados favorables y mejoras significativas en las tres dimensiones del DF: actividades básicas, fuerza y equilibrio y velocidad de marcha en pacientes con EP del CEMENA.

\section{REFERENCIAS}

1. Micheli F. Enfermedad de Parkinson y Trastornos relacionados. 2a ed. Buenos Aires: Edit. Médica Panamericana; 2011.

2. Borrione P, Tranchita E, Sansone P, Parisi A. Effects of physical activity in Parkinson's disease: A new tool for rehabilitation. World J Methodol. [Internet] 2014; 4(3): 133-143 [Consultado 2019 Nov 8] Disponible en: doi: 10.5662/wjm.v4.i3.133.
3. Hurtado F, Cárdenas MA, Cárdenas $F$, León LA. La Enfermedad de Parkinson: Etiología, Tratamientos y Factores Preventivos. Univ. Psychol. [Internet] 2016; 15(5): 1-26 [Consultado 2019 Nov 8] Disponible en: http://www.scielo.org.co/pdf/rups/v15nspe5/ v15nspe5a12.pdf

4. Pérez C, Vargas C, Silva J, Cortes S. Comportamiento de la mortalidad por la Enfermedad de Parkinson en Chile en el periodo 1990-2009. Acta Neurol Colomb. 2014; 30(2): 97-102.

5. Rodríguez-Violente $M$, Villar-Velarde $A$, Valencia-Ramos C, Cervantes-Arriaga A. Características epidemiológicas de pacientes con Enfermedad de Parkinson de un hospital de referencia en México. Arch Neurocien. 2011; 16(2):64-68.

6. Bleton JP, Ziégler M. Rehabilitación de la enfermedad de Parkinson. EMC - Kinesiterapia - Medicina Física. 2012; 33(1): 1-15.

7. Torres L, Mori N, Cuentas M, Dominguez J, Mendoza $M$, Mendoza $M$, et al. Prevalencia de la enfermedad de Parkinson. Un estudio puerta a puerta en cinco distritos de Ulcumayo - Junín, Perú. Diagnóstico. 2008; 47(4): 150-56.

8. Cano de la Cuerda R, Macías-Jiménez Al, CrespoSánchez V, M. Morales-Cabezas M. Escala de valoración y tratamiento fisioterapéutico en la enfermedad de Parkinson. Fisioterapia. 2004; 26(4): 201-210.

9. Behari M, Srivastava, AK, Pandey RM. Quality of life in patients with Parkinson's disease. Parkinsonism \& Related Disorders, 2005; 11(4): 221-226.

10. Kulisevsky MR, Luquin JM, Arbelo JA, Burguera F, Carrillo A, Castro J et al. Enfermedad de Parkinson Avanzada: Características clínicas y tratamiento. Parte II. Neurología. 2013; 28(9): 558-583.

11. Ellis TD, Cavanaugh JT, DeAngelis T, Hendron $K$, Thomas CA, Saint-Hilaire $M$, et al. Comparartive Effectiveness of mHealth-Supported Exercise Compared With Exercise Alone for People With Parkinson Disease: Randomized Controlled Pilot Study. Phys Ther. [Internet] 2019; 99(2): 203-216 [Consultado 2019 Nov 8] Disponible en: 10.1093/ptj/pzy131.

12. Hernández R, Fernández $C$, Baptista P. Metodología de la investigación. 6a ed. México: Editorial McGraw-HillInteramericana; 2014.

13. Seco-Calvo J, Gago-Fernández I. Eficacia de un programa intensivo y continuado de fisioterapia para la mejoría clínica en pacientes con enfermedad de Parkinson. Fisioterapia [Internet] 2010; 32(5):208-216 [Consultado 2019 Nov 8] Disponible en: doi: 10.1016/j.ft.2010.06.002

14. Tipos de estudios clínico epidemiológicos [Internet] Alicante: Universidad de Alicante; 1995 [Consultado 2019 May 17) Disponible en: https://www.fisterra.com/ mbe/investiga/6tipos_estudios/6tipos_estudios.asp

15. Navarro-Petemella FM, Marcon SS. Calidad de vida de las personas con Enfermedad de Parkinson y su relación con la evolución en el tiempo y la gravedad de la enfermedad. Rev Latino Am Enfermagen. [Internet] 2012; 20(2):1-8 [Consultado 2019 Dic 9) Disponible en: www.eerp.usp.br/rlae

16. Seco-Calvo J, Gago-Fernández I, Cano-de-la-Cuerda R, Fernández-de-las-Peñas C. Efectividad de los estímulos sensoriales sobre los trastornos de la marcha en pacientes con enfermedad de Parkinson. Estudio piloto. 
Fisioterapia [Internet] 2012; 34(1): 4-10 [Consultado 2019 Nov 8] Disponible en: doi:10.1016/j.ft.2011.07.009.

17. Abizanda P, López-Torres J, Romero L, Sánchez PM, García I, Esquinas JL. Valores normativos de instrumentos de valoración funcional en ancianos españoles: estudio FRADEA. Atencion Primaria [Internet] 2012; 44(3): 162171 [Consultado 2019 Ago 21] Disponible en: https:// doi.org/10.1016/j.aprim.2011.02.007

18. Abizanda $\mathrm{P}$, Romero L. Innovación en la valoración funcional. Rev Esp Geriatr Gerontol [Internet] 2006; 41(S1): 27-35 [Consultado Año Mes Día; ej. 2020 Dic 20] Disponible en: doi: 10.1016/S0210-5705(09)71003-9

19. Benavides R, García G, Fernández O, Rodríguez B, Ariza J. Condición física, nivel de actividad física, y capacidad funcional en el adulto mayor: Instrumentos para su cuantificación. Rev U.D.C.A. Act. \& Div. Cient. [Internet] 2017; 20(2): 255-265 [Consultado 2019 Ene 18] Disponible en: http://www.scielo.org.co/pdf/rudca/ v20n2/v20n2a04.pdf

20. Guralnik JM, Ferrucci L, Simonsick EM, Salive ME, Wallace $\mathrm{RB}$, Lower-extremity function in persons over the age of 70 years as a predictor of subsequent disability. NEngl J Med 1995; 332: 556-561.

21. Declaración de Helsinki de la Asamblea Médico Mundial - Principios éticos para las investigaciones médicas en seres humanos [Internet] Francia: WMA The World Medical Association; 2017 [Consultado 2019 Mar 27] Disponible en: https://www.wma.net/es/policies-post/ declaracion-de-helsinki-de-la-amm-principios-eticospara-las-investigaciones-medicas-en-seres-humanos/

22. Gillies G, Pienaar I, Vohra S, Qamhawi Z. Sex differences in Parkinson's disease. Front Neuroendocrinol. 2014; 35(3):370-384. doi: 10.1016/J.Yfrne.2014.02.002.

23. Soundy A, Collett J, Lawrie S, Coe S, Roberts H, Hu M, et al. A Qualitative study on the impact of firts steps. A peer-led educational intervention forpeople newly diagnosed with Parkinson's disease. Behav Sci (basel) [Internet] 2019; 9(10):107 [Consultado 2019 Nov 8] Disponible en: doi: 10.3390/bs9100107

24. Scollo S, Emanuel F, Crimi D, Rodríguez-Quiroga S, Christie C, Díaz-Aragunda V, et al. Progresión clínica de la enfermedad de Parkinson: análisis retrospectivo en un consultorio especializado en trastorno del movimiento. Neurologia Argentina. 2016; 8(4): 258-262.

25. Jurado-Coronel JC, Cabezas R, Ávila-Rodríguez MF, Echeverría V, García-Segura LM, Barreto GE. Sex differences in Parkinson's disease: Features on clinical symptoms, treatment outcome,sexual hormones and geenetics. Front Neuroendocrinol. [Internet] 2018; 50: 18-30 10 [Consultado 2019 Nov 8] Disponible en: doi: 10.1016/j.yfrne.2017.09.002

26. Ashburn H, Pickering R, McIntosh E, Hulbert S, Rochester $L$, Roberts $H C$, et al. Exercise and strategy based physiotherapy delivered intervention for preventing repeat falls in people with Parkinson's: The PDSAFE RCT. Healt Technol Assess [Internet] 2019; 23(36): 1-150 [Consultado 2019 Nov 8] Disponible en: doi: 10.3310/ hta23360.

27. Cersosimo M, Raina G, Pellene L, Micheli FE, Calandra CR, Mailoa R. Weigth loss in Parkinson's disease: The relationship with motor symptoms and disease progression. BioMed research International [Internet]
2018; 1-6 [Consultado 2019 Nov 8] Disponible en: https://doi.org/10.1155/2018/9642524

28. Hunter H, Lovegrove C, Haas B, Freeman J, Gunn $\mathrm{H}$. Experiences of people with Parkinson's disease and their views on physical activity interventions: a qualitative systematic review. JBI Database System Rev Implement Rep. [Internet] 2019; 17(4): 548-613. [Consultado 2019 Nov 8] Disponible en: doi: 10.11124/ JBISRIR-2017-003901

29. Hashimoto $H$, Takabatake $S$, Miyaguchi $H$, Nakanishi $H$, Naitouu Y. Effects of dance on motor functions, cognitive functions, and mental symptoms of Parkinson's disease: a quasi-randomized pilot trial. Complement Ther Med. [Internet] 2015; 23(2): 210-9 [Consultado 2019 Nov 8] Disponible en: doi: 10.1016/j.ctim.2015.01.010

30. Vaartio-Rajalin H, Rahuala A, Fagerstrom L. Personcentered home-based rehabilitation for persons with Parkinson's disease: A scoping review. Int J Nurs Stud. [Internet] 2019; 99: 103395. [Consultado 2019 Nov 8] Disponible en: doi: 10.1016/j.ijnurstu.2019.103395

31. States R, Sweeny T, Rossi A, Spiever D, Salem R. Physical functioning after 1,3 , and 5 years of exercise among people with Parkinson's disease: A longitudinal observational study. J Geariatry Phys Therp. [Internet] 2017; 40(3): 127-134. [Consultado 2019 Nov 8] Disponible en: doi: 10.1519/JPT.0000000000000084

32. Franciotta $M$, Maestri $R$, Ortelli $P$, Ferrazzoli $D$, Mastalli F, Frazzitta G. Occupational Therapy for Parkinsonian Patients: A Retrospective Study. Parkinsons Dis. [Internet] 2019; 4561830 [Consultado 2019 Nov 8] Disponible en: doi: 10.1155/2019/4561830

33. Ferrazzoli D, Ortelli P, Zivi I, Cian V, Urso E, Ghilardi MF et al. Efficacy of intensive multidisciplinary rehabilitation in Parkinson's disease: A randomised controlled study. Journal of Neurology, Neurosurgery and Psychiatry. [Internet] 2018; 89(8): 828-835 [Consultado 2019 Nov 8] Disponible en: doi: http://dx.doi.org/10.1136/jnnp2017-316437

34. Ortelli P, Ferrazoli D, Bera R, Caremani L, Giladi N, Maestri $R$ et al. Effectiveness of a goal-based intensive rehabilitation in parkinsonian patients in advanced satges of disease. Journal of Parkinson's Disease [Internet] 2018; 8(1): 113-119 [Consultado 2019 Nov 8) Disponible en: doi: 10.3233/JPD-171247

35. Tickle-Degnen $L$, Ellis T, Saint-Hilaire $M H$, Thomas CA, Wagenaar RC, et al. Self-management rehabilitation and health-related quality of life in Parkinson's disease: a randomized controlled trial. Mov Disord. [Internet] 2010; 25: 194-204. [Consultado 2019 Nov 8] Disponible en: doi: $10.1002 / m d s .22940$

36. Reynolds GO, Otto M, Ellis TD, Cronin-Golomb A. The therapeutic potential of exercise to improve moud, cognition and sleep in Parkinson's disease. Mov Disord. [Internet] 2016; 31(1): 23-38. [Consultado 2019 Nov 8] Disponible en: doi: $10.1002 / \mathrm{mds} .26484$

37. Gobbi LT, Oliveira-Ferreira MD, Caetano MJ, LiraniSilva E, Barbieri FA, Stella F. Exercise programs improve mobility and balance in people with Parkinson's disease. Parkinsonism Relat Disord. [Internet] 2009; 15(suppl 3): s49-52 [Consultado 2019 Nov 8]Disponible en: doi: $10.1016 / \mathrm{S} 1353-8020(09) 70780-1$ 